\title{
CORRECTIONS
}

\section{The truth about sports drinks}

Corrections to the Feature "Forty years of sports performance research and little insight gained" (BMJ 2012;345:e4797), by Carl Heneghan and colleagues, have necessitated a correction to this accompanying Feature by Deborah Cohen (BMJ 2012;345:e4737, doi:10.1136/bmj.e4737). The "What the research found" box contains two errors where it cites the former article. Firstly, in the third paragraph, "Small sample sizes limit the applicability of results," the first sentence should have begun by noting that "Only one of the 105 [not "106"] studies . . . exceeded the acceptable target for a small study of 100 participants per group." Secondly, the statement in the fifth paragraph that "most studies (76\%) were low in quality" should have instead said $70 \%$.

Cite this as: BMJ 2012;345:e6096

๑ BMJ Publishing Group Ltd 2012 